\title{
Differential metabolic markers associated with primary open-angle glaucoma and cataract in human aqueous humor
}

\author{
Chen-Wei Pan ${ }^{1 *+} \mathbb{B}$, Chaofu Ke ${ }^{1 \dagger}$, Qin Chen², Yi-Jin Tao ${ }^{3}$, Xu Zha $^{4}$, Yuan-Ping Zhang ${ }^{4}$ and Hua Zhong ${ }^{3 *}$
}

\begin{abstract}
Background: We aimed to identify metabolic biomarkers and investigate the metabolic alterations in relation to primary open-angle glaucoma (POAG) and cataract in human aqueous humor.

Methods: Sixteen POAG patients undergoing surgical treatments and 24 patients undergoing cataract surgeries were included in this case-control study. We performed the metabolomic analysis of aqueous humor samples using a non-targeted gas chromatography coupled to time-of-flight mass spectrometer. The area under the receiver operating characteristic curve (AUC) was computed to assess the discrimination capacities of each metabolite marker. Databases including the Kyoto Encyclopedia of Genes and Genomes (KEGG) and MetaboAnalyst were utilized to search for the potential pathways of metabolites.

Results: Aqueous humor metabolomic profiles could well distinguish POAG from controls. Fourteen metabolic biomarkers were identified as potential aqueous humor biomarkers for POAG, yielding AUC values from 0.62 to 0.86. In pathway analysis, Biotin metabolism was highly impacted, implying that these metabolic markers play important roles in the regulation of this pathway.

Conclusions: This study identified valuable metabolic biomarkers and pathways that may facilitate an improved understanding of the POAG pathogenesis. The finding holds translational value in the development of new therapeutic measures for POAG.
\end{abstract}

Keywords: Open-angle glaucoma, Cataract, Epidemiology, Metabolomics

\section{Background}

Primary open-angle glaucoma (POAG) is the most common subtype of glaucoma and the major cause of irreversible blindness throughout the world [1]. Although numerous studies have identified several important ocular risk factors for POAG such as increased intraocular pressure (IOP) [2,

\footnotetext{
* Correspondence: pcwonly@gmail.com; zhoculist@163.com

${ }^{\dagger}$ Chen-Wei Pan and Chaofu Ke contributed equally to the work presented here and therefore should be considered equivalent authors.

'School of Public Health, Medical College of Soochow University, 199 Ren Ai Road, Suzhou 215123, China

${ }^{3}$ Department of Ophthalmology, the First Affiliated Hospital of Kunming Medical University, No. 295 Xichang Road, Kunming 650032, China

Full list of author information is available at the end of the article
}

3], myopic refractive errors [4], larger optic disc size [5, 6] and thinner central corneal thickness [7, 8], these findings are limited in understanding the pathophysiology of POAG. Further knowledge regarding the pathophysiology might help to create new drug development research lines and expand current therapeutic targets for POAG. In current clinical practice, the treatment strategy of POAG mainly relies on IOP-lowering medications or surgeries. Although increased IOP is widely accepted to be the primary predictor for POAG, glaucomatous neuropathy is still observed in some patients with normal or even lower-than-normal IOPs, suggesting that other mechanisms exist in the pathophysiology of POAG. 
Metabolomics is a widely used technology to assess biomarkers for diseases and provide molecular information regarding disease phenotype since metabolites are the ultimate product of gene, mRNA and protein activities [9]. Variations in the metabolome represent the interplay of genetic and environmental factors and are in relation to disease states, which may shed some lights in mechanism and pathophysiology of the disease [10]. With regard to eye diseases, metabolomics has been successfully used in identifying the metabolic signatures of diabetic retinopathy [11]. However, there were less studies focusing on POAG, especially in human participants. A previous analysis comparing plasma metabolic signatures as measured by mass spectrometry observed significant differences in some specific metabolic processes such as palmitoylcarnitine, sphingolipids, vitamin Drelated compounds, and steroid precursors between POAG patients and healthy controls [12]. These differences observed in metabolome might be linked to mitochondrial dysfunction and energy metabolism changes [12]. However, we believe that aqueous humor samples are more sensitive to detect biomarkers in glaucomatous eyes, which may provide novel insights into more new pathogenic pathways for this ocular condition. To the best of our knowledge, few studies have focused on the aqueous humor metabolite markers of POAG in human beings. To address this gap, we performed a clinicalbased case-control study and aimed to identify novel metabolite markers of POAG in human aqueous humor samples.

\section{Methods}

\section{Study design and participants}

A clinical-based case-control study was conducted on patients in two tertiary hospitals in China including the First Affiliated Hospital of Kunming Medical University and the First Affiliated Hospital with Nanjing Medical University. Two ophthalmologists (Qin Chen and Hua Zhong) collected aqueous humor samples during the surgical treatment in consecutive samples of 40 POAG and 40 cataract patients $(20$ cases and 20 controls in each hospital). Considering that no sample size calculation rationales are available for metabonomic study at current stage, the sample size was determined based on previous published literatures on metabonomic studies of aqueous humor samples as well as our available resources. In this study, cases were POAG participants who undertook surgical treatment and were free of cataract. POAG patients were considered to be free of cataract if the nuclear opalescence or nuclear color was less than 4, the cortical cataract score was less than 2, and the posterior subcapsular cataract score was less than 2 in the affected eye based on the Lens Opacities Classification System (LOCS) III [13]. Controls were participants who undertook cataract surgeries. The samples of aqueous humor (at least $20 \mu \mathrm{L}$ ) were extracted for each participant during the surgical treatment for both cases and controls. Aqueous humor samples were stored in a freezer at the temperatures of $-80^{\circ} \mathrm{C}$ immediately after it was extracted during the surgical treatment.

The study was conducted following the tenets of the Helsinki Declaration and was approved by the Institutional Review Board of the Kunming Medical University. All patients included in the study provided written informed consent for aqueous humor samples to be extracted.

\section{Metabonomic profiling and data processing}

First, derivatization of the samples was performed according to the protocols reported in a previous study [14]. Then, all samples were analyzed by gas chromatograph system coupled with a Pegasus HT time-of-flight mass spectrometer (GC-TOF-MS). GC-TOF-MS analysis was performed using an Agilent 7890 gas chromatograph system coupled with a Pegasus HT time-offlight mass spectrometer. A $1 \mu \mathrm{L}$ aliquot of the analyte was injected in a splitless mode. Helium was used as the carrier gas, the front inlet purge flow was $3 \mathrm{~mL}$ per minute, and the gas flow rate through the column was 1 $\mathrm{mL}$ per minute. The initial temperature was kept at $50{ }^{\circ} \mathrm{C}$ for $1 \mathrm{~min}$, then raised to $310^{\circ} \mathrm{C}$ at a rate of $20^{\circ} \mathrm{C}$ per min and was kept for $6 \mathrm{~min}$ at $310^{\circ} \mathrm{C}$. The injection, transfer line, and ion source temperatures were $280^{\circ} \mathrm{C}$, $280^{\circ} \mathrm{C}$, and $250{ }^{\circ} \mathrm{C}$, respectively.

Chroma TOF 4.3X software was used for raw peaks exacting, data baselines filtering and calibration of the baseline, peak alignment, deconvolution analysis, peak identification and integration of the peak area. Mass spectrum match and retention index match were considered in metabolites identification. We removed peaks detected in less than $50 \%$ of quality control (QC) samples or relative standard deviation (RSD) more than $30 \%$ in QC samples.

\section{Statistical analyses}

First of all, peaks could be left through interquartile range denoising method. Then the missing values of raw data were filled up by half of the minimum value. A multivariate analysis was performed using the SIMCA14.1 software package (V14.1, Sartorius Stedim Data Analytics AB, Umea, Sweden). An unsupervised model of principal component analysis (PCA) with unit variance scaling was applied to show the distribution of origin data [15]. In order to obtain a higher level of group separation and get a better understanding of variables responsible for classification, supervised orthogonal projections to latent structures-discriminate analysis 
(OPLS-DA) were applied [16]. To refine this analysis, the first principal component of variable importance in the projection (VIP) was obtained. The VIP values exceeding 1 were first selected as changed metabolites. In addition, these selected metabolites were further validated at a critical $P$ value of 0.05 using two sided student's t-test. The area under the receiver operating characteristic curve (AUC) was computed to assess the discrimination capacities of each metabolite marker. Databases including the Kyoto Encyclopedia of Genes and Genomes (KEGG) [17] (http://www.genome.jp/kegg/) and MetaboAnalyst [18](http://www.metaboanalyst.ca/) were utilized to search for the pathways of metabolites.

\section{Results}

\section{Characteristics of the participants}

In the end of the study, 16 patients with POAG (40\%) undergoing surgical treatments and 24 patients $(60 \%)$ undergoing cataract surgeries from the two hospitals provided written informed consent for their aqueous humor samples to be taken for research purpose. Thus, the ratio for cases vs. controls was 1:1.5. The mean age was 72.5 years in participants with POAG (cases) and 74.2 years in participants with cataract (controls) and the difference was not statistically significant $(P=0.13)$. Women accounted for $54 \%$ of the study sample in cases and $62 \%$ in controls. No preoperative medications were used for all participants.

\section{Aqueous humor metabolic profiles}

The PCA score plot showed that the QC samples were tightly clustered, supporting the robustness of the metabolic profiling platform (data not shown). The supervised OPLS-DA model was established to understand the holistic metabolic differences between POAG patients and controls. As shown in the OPLS-DA score plot, excellent separation between POAG patients and controls could be achieved (Fig. 1). The validation plot strongly supported the validity of the model, as all permuted R2 and Q2 values on the left were lower than the original points on the right (Fig. 2).

\section{Identification of potential biomarkers}

Following the successful establishment of the OPLS-DA model, potential metabolic biomarkers were selected using the criteria of VIP or more than 1.0 and $P$ value of less than 0.05 . Finally, 14 metabolites were successfully selected and identified as potential biomarkers of POAG compared with controls (Table 1). Compared with controls, 6 metabolites were found to be decreased in those with POAG compared with cataract, including Biotin, Glucose-1-phosphate, Methylmalonic acid, N-cyclohexylformamide 1, Sorbitol, and

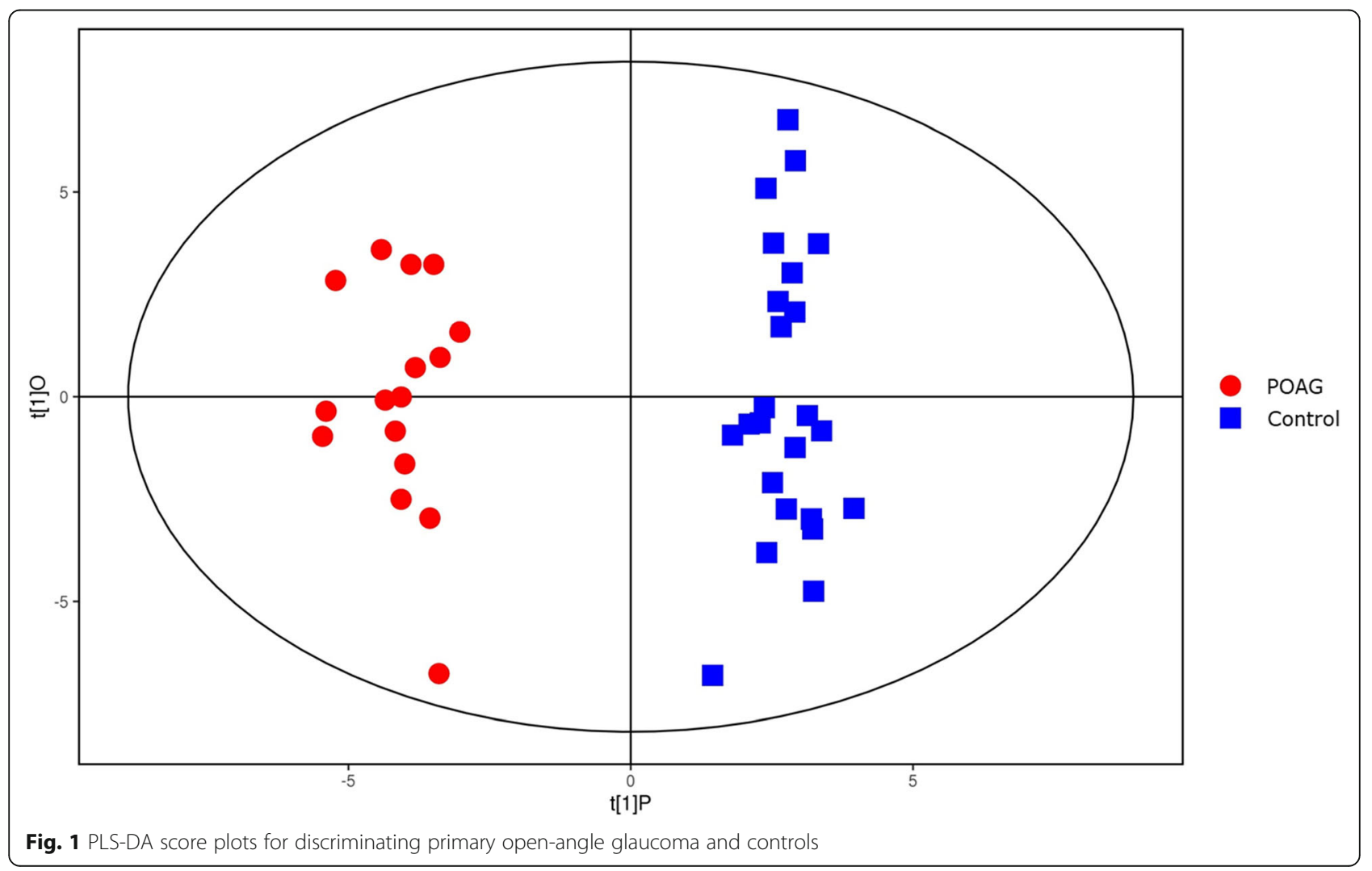




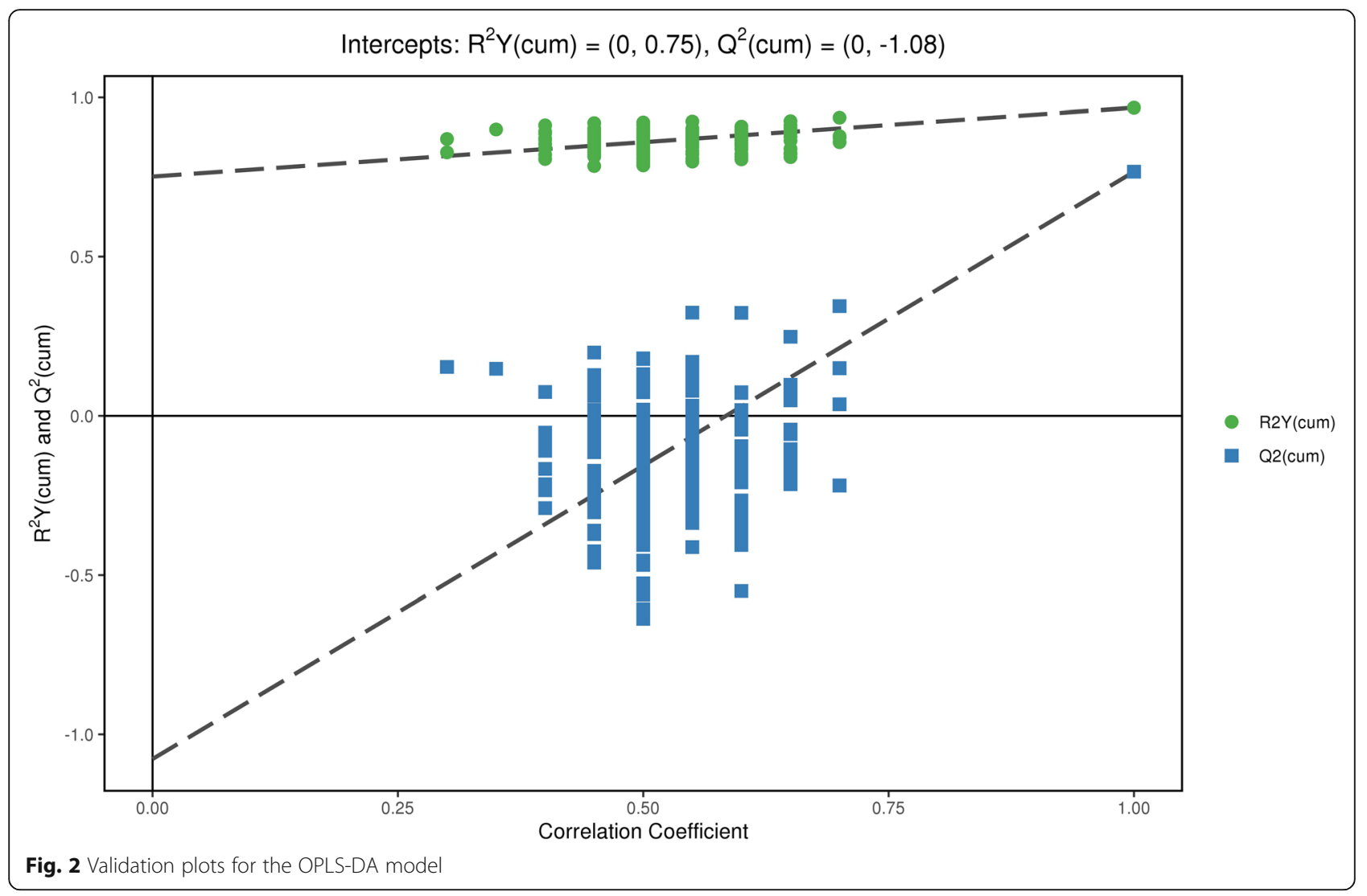

Table 1 Potential metabolic biomarkers identified for primary open-angle glaucoma in aqueous humor samples

\begin{tabular}{|c|c|c|c|c|c|}
\hline $\mathrm{ID}$ & Metabolite & VIP & $P$ value & Up/Down Regulation & $A \cup C$ \\
\hline 1 & Glucose-1-phosphate & 1.57 & 0.04 & Down & 0.64 \\
\hline 2 & Methylmalonic acid & 1.16 & 0.04 & Down & 0.68 \\
\hline 3 & Spermidine 2 & 1.43 & 0.19 & Down & 0.69 \\
\hline 4 & N-cyclohexylformamide 1 & 1.57 & 0.01 & Down & 0.73 \\
\hline 5 & Sorbitol & 1.67 & 0.02 & Down & 0.74 \\
\hline 6 & Biotin & 1.13 & 0.05 & Down & 0.67 \\
\hline 7 & Pelargonic acid & 1.02 & 0.01 & Up & 0.75 \\
\hline 8 & 2-mercaptoethanesulfonic acid 2 & 2.67 & $<0.001$ & Up & 0.83 \\
\hline 9 & Galactose 1 & 3.01 & $<0.001$ & Up & 0.86 \\
\hline 10 & Mannose 1 & 2.43 & $<0.001$ & Up & 0.80 \\
\hline 11 & D-erythronolactone 2 & 1.27 & 0.05 & Up & 0.62 \\
\hline 12 & Dehydroascorbic Acid 2 & 2.77 & $<0.001$ & Up & 0.86 \\
\hline 13 & Ribitol & 2.49 & $<0.001$ & Up & 0.78 \\
\hline 14 & D-Talose 1 & 3.26 & $<0.001$ & Up & 0.85 \\
\hline
\end{tabular}

VIP Variable importance in the projection; AUC Area under the receiver operating characteristic curve 


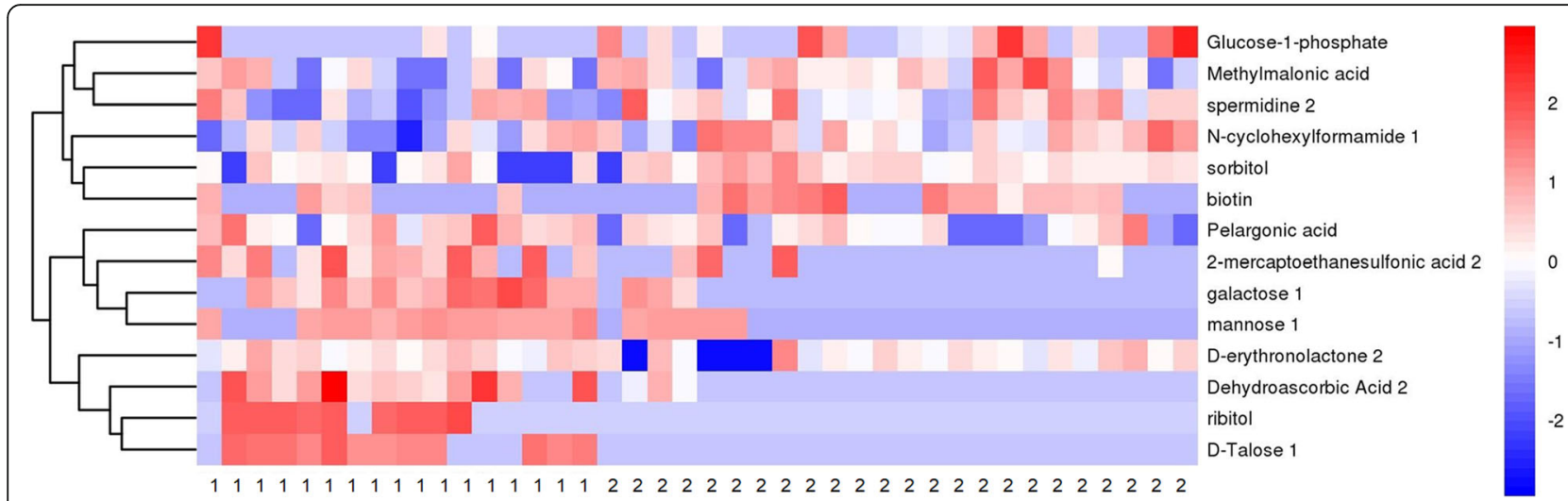

Fig. 3 A heatmap showing the concentrations of 14 metabolite markers between primary open-angle glaucoma and controls (1: primary openangle glaucoma, 2: controls)

Spermidine 2. In contrast, 8 metabolites, including 2mercaptoethanesulfonic acid 2, D-erythronolactone 2, DTalose 1, Dehydroascorbic Acid 2, Galactose 1, Mannose 1, Pelargonic acid and Ribitol, were found to be increased in participants with POAG compared with controls (Fig. 3). Those metabolite markers showed the potential to discriminate between POAG and controls, with AUC values ranging from 0.62 to 0.86 (Table 1 ).

\section{Pathway analysis for potential biomarkers}

Pathway analysis, including enrichment analysis and pathway topology analysis, was further performed to understand the metabolic pathways that these potential biomarkers are involved in. A total of 5 pathways were significantly enriched at the significance level of 0.10 , namely Biotin metabolism; Beta-Alanine metabolism; Glutathione metabolism; Folate biosynthesis; and Arginine and Proline metabolism (Fig. 4). Especially, Biotin metabolism was highly impacted, implying that these metabolic markers play important roles in the regulation of this pathway.

\section{Discussion}

In clinical practice, POAG remains a poorly understood condition with limited therapeutic options. Therefore, there is a pressing need to develop personalized

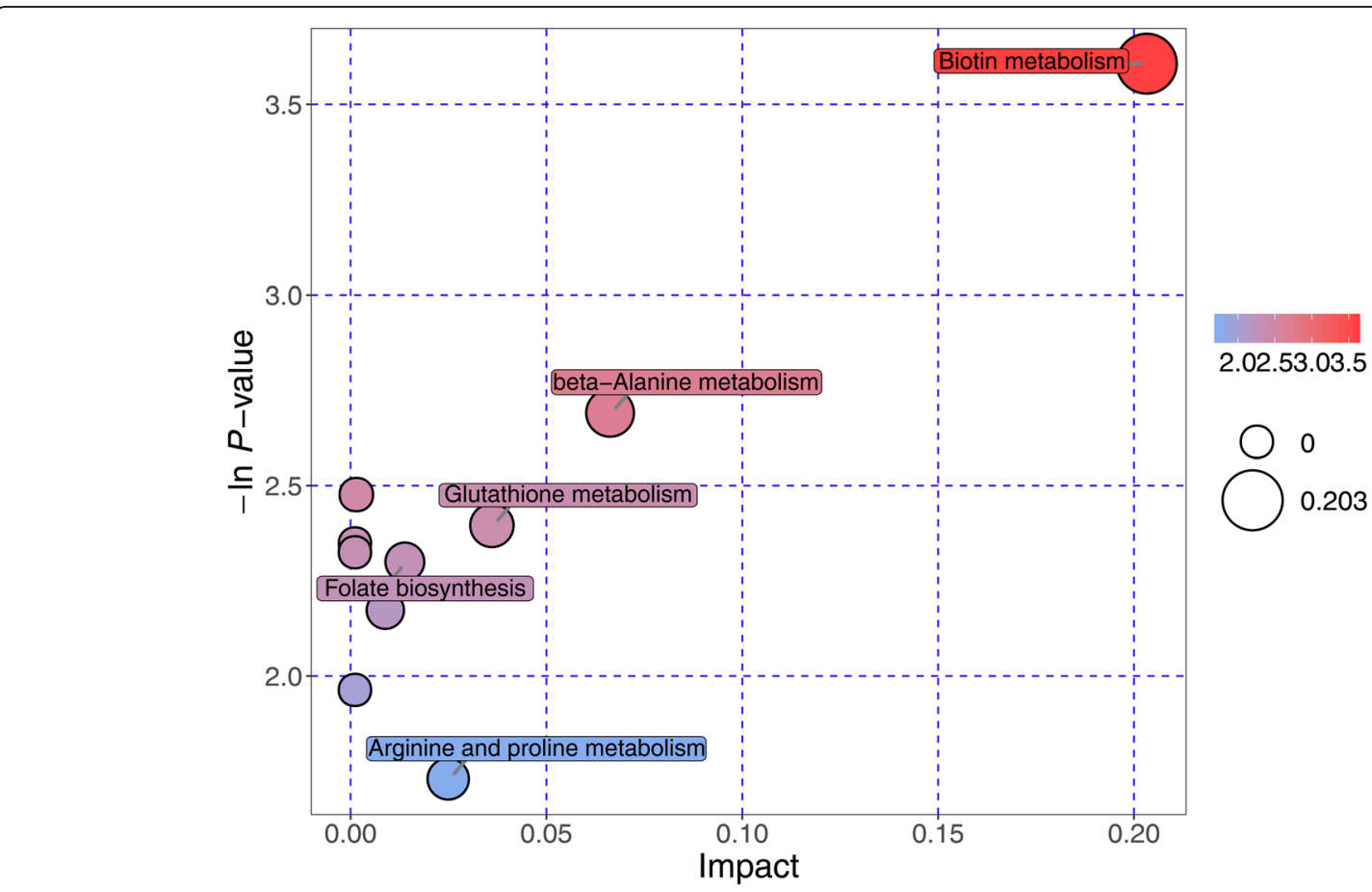

Fig. 4 Enrichment analysis and pathway topology analysis for potential metabolic biomarkers of primary open-angle glaucoma 
approach to guide clinical management. In this study, we systematically explored the metabolic differences in aqueous humor samples between patients with POAG and controls of patients with cataract. We identified fourteen metabolites as possible biomarkers in aqueous humor samples that had the potentials to distinguish POAG patients from controls. The identification of novel metabolite markers for POAG in human aqueous humor provides insights into potential new pathogenic pathways for this vision-threatening condition and could potentially lead to new drug development research lines.

Previous animal and human studies have provided some preliminary results on metabolites associated with glaucoma. For example, an experimental study found that metabolites in relation to osmotic stress, oxidative stress and glucose metabolism were related to the retinal ganglion cell death in rats, which is regarded as the common mechanism for glaucoma [19]. Another mouse model of chronic glaucoma identified unique profiles of sphingolipid and ceramide species between normotensive and hypertensive aqueous humor and found that sphingosine- and sphinganine-1-phosphates greatly increased in hypertensive mice [20]. With regard to the studies on humans, an untargeted plasma metabolomic study observed increased levels of palmitoylcarnitine; putatively-identified sphingosine, sphinganine, and decreased levels of sphingosine-1-phosphate in patients with POAG [12]. To the best of our knowledge, few studies have explored the POAG-associated metabolites in human aqueous humor samples. The analysis of metabolites in human aqueous humor may reveal novel potential biomarkers of POAG with greater sensitivity and specificity which might be detected in blood samples. We identified 14 metabolite markers in human aqueous humor which might potentially distinguish POAG from cataract. We also searched public databases such as KEGG [17] and MetaboAnalyst [18] and other published literatures, trying to find possible metabolic pathways for POAG observed in this study.

We found that Biotin metabolism was highly impacted in the pathway analysis, implying that Biotin may play major roles in POAG pathophysiology. Biotin was reported be oxidized into the retina [21]. It was also indicated that in vivo administration of biotin to early embryonic chick eyes at moderately elevated levels induced malformations, mainly affecting lens structures [22]. As the controls in this study were patients undergoing cataract surgeries whose lens structures might have been malformed compared with cases free of cataract, it is reasonable that the levels of Biotin is lowers in POAG patients observed in this study.

The phenomenon that Methylmalonic acid was reduced in POAG patients compared with controls probably suggested increased oxidative damage may play a major pathophysiological role in POAG [23].. Methylmalonic acid was reported to be able to provoke oxidative damage and compromise antioxidant defenses in nerve terminal and striatum [24]. According to the results of a recent meta-analysis including 22 case-control studies with 1614 with glaucoma and 1319 healthy controls, some biomarkers increased in glaucomatous aqueous humor such as superoxide dismutase and glutathione peroxidase. In addition, high levels of Methylmalonic acid were also found to increase the risk of optic neuropathy [25-27].

The level of Ribitol in aqueous humor was found to be higher in POAG compared with cataract in our study. Increased level of Ribitol was reported to play a role in prevention of cataract formation and act as a cofactor for glutathione reductase, which is linked to cataract formation by decreased glutathione levels in the lenses. On the other hand, Ribitol deficiency is implicated in the formation of cataracts due to the concentration of reduced glutathione in the lens and its ability to protect the tissue from oxidative damage [28]. Thus, our finding is consistent with previous studies.

The level of Sorbitol was expected to be lower in POAG patients as compared with those with cataract. It is well established that diabetes is a major risk factor cataract [29]. Sorbitol is a key marker in osmotic stress. Lens fiber cell resulting from excessive accumulation of sorbitol has been proposed as a possible mechanism in cataract formation in diabetic patients. A previous study reported a strong relationship between the abundance of polyol pathway metabolites sorbitol and blood glucose levels in lenses extracted from diabetic patients [30].

Mannose was found to be increased in POAG patients compared with cataracts. Mannose is a simple sugar but have a complex health effect on different parts of the body including the eyes. A previous study have indicated that an upregulation of the lectin pathway-associated mannose-serine-protease- 2 was observed in the optic nerves of the optic nerve homogenate antigen group [31], which supports the finding in the current study.

We have to acknowledge some limitations of this study. One major limitation was the small sample size, which may have prevented changes in certain metabolites from being apparent. In addition, some sources of bias such as differences in the time of day of sample collection may have distorted the findings. Particularly, intake of drugs such as IOP-lowering medications in patients with POAG may have potentially altered the metabolome distributions. However, it is unlikely to control the effect of IOP-lowering medications between cases and controls in this study. Furthermore, the control group was patients undergoing cataract surgery rather than "healthy" individuals, and the selection of control group might have distorted the findings as cataract 
should have its own distinct metabolic profiling which should be different from POAG. It is ideal if we could obtain aqueous humor samples in healthy people without eye diseases. Finally, metabolic pathways of some metabolites identified in this study could not be founded in public databases or published literatures. Whether they are true biomarkers for POAG warrants further clarifications.

\section{Conclusions}

This study investigated the metabolic markers associated with POAG in human aqueous humor samples. As a result, 14 metabolites were identified as potential biomarkers that could discriminate between patients with POAG and controls. Biotin metabolism was highly impacted in pathway analysis and may play important roles in the regulation of this pathway. Overall, this study provided new clues in the disease pathogenesis of POAG. Validation of the results is warranted in other studies.

\section{Abbreviations}

POAG: Primary open-angle glaucoma; IOP: Intraocular pressure; LOSC: Lens opacities classification system; QC: Quality control; RSD: Relative standard deviation; PCA: Principal component analysis; VIP: Variable importance in the projection; AUC: Receiver operating characteristic curve

\section{Acknowledgements}

Not Applicable.

\section{Authors' contributions}

CWP and $H Z$ designed the study. QC, YJT, XZ, YPZ and $H Z$ performed the research. CWP and CK analyzed the data and wrote the paper. CWP and HZ supervised the study. All authors have read and approved the manuscript.

\section{Funding}

This study was funded by the National Natural Science Foundation of China under grant no. 81973061, grant no. 81970800 and grant no. 81860173 . The funders had no role in study design, data collection and analysis, decision to publish, or preparation of the manuscript.

\section{Availability of data and materials}

The datasets analyzed in this study are available from the corresponding author (Chen-Wei Pan, pcwonly@gmail.com) upon reasonable request.

\section{Ethics approval and consent to participate}

Ethics committee approval was obtained from the Institutional Review Board of Kunming Medical University. We carried out the study according to the tenets of the Declaration of Helsinki involving human participants and the approved guidelines. All patients included in the study provided written informed consent for aqueous humor samples to be extracted.

\section{Consent for publication}

Not applicable.

\section{Competing interests}

The authors declare that they have no competing interests.

\section{Author details}

'School of Public Health, Medical College of Soochow University, 199 Ren Ai Road, Suzhou 215123, China. 'Department of Ophthalmology, the First Affiliated Hospital with Nanjing Medical University, Nanjing, China. ${ }^{3}$ Department of Ophthalmology, the First Affiliated Hospital of Kunming Medical University, No. 295 Xichang Road, Kunming 650032, China. ${ }^{4}$ Department of Ophthalmology, the Second Affiliated Hospital of Kunming Medical University, Kunming, China.
Received: 14 January 2020 Accepted: 27 April 2020

Published online: 06 May 2020

\section{References}

1. Tham YC, Li X, Wong TY, Quigley HA, Aung T, Cheng CY. Global prevalence of glaucoma and projections of glaucoma burden through 2040: a systematic review and meta-analysis. Ophthalmology. 2014;121(11):2081-90.

2. Wang D, Huang W, Li Y, Zheng Y, Foster PJ, Congdon N, He M. Intraocular pressure, central corneal thickness, and glaucoma in chinese adults: the liwan eye study. Am J Ophthalmol. 2011;152(3):454-62 e451.

3. Pan CW, Yang WY, Hu DN, Xu JG, Niu ZQ, Yuan YS, Yu MB, Zhong H. Longitudinal cohort study on the incidence of primary open-angle Glaucoma in Bai Chinese. Am J Ophthalmol. 2017:176:127-33.

4. Marcus MW, de Vries MM, Junoy Montolio FG, Jansonius NM. Myopia as a risk factor for open-angle glaucoma: a systematic review and meta-analysis. Ophthalmology. 2011;118(10):1989-94 e1982.

5. Healey PR, Mitchell P. Optic disk size in open-angle glaucoma: the Blue Mountains eye study. Am J Ophthalmol. 1999;128(4):515-7.

6. Jonas JB, Xu L, Zhang L, Wang Y, Wang Y. Optic disk size in chronic glaucoma: the Beijing eye study. Am J Ophthalmol. 2006;142(1):168-70.

7. Day AC, Machin D, Aung T, Gazzard G, Husain R, Chew PT, Khaw PT, Seah SK, Foster PJ. Central corneal thickness and glaucoma in east Asian people. Invest Ophthalmol Vis Sci. 2011;52(11):8407-12.

8. Herndon LW, Weizer JS, Stinnett SS. Central corneal thickness as a risk factor for advanced glaucoma damage. Arch Ophthalmol. 2004;122(1):17-21.

9. Kell DB, Goodacre R. Metabolomics and systems pharmacology: why and how to model the human metabolic network for drug discovery. Drug Discov Today. 2014;19(2):171-82.

10. Holmes E, Wilson ID, Nicholson JK. Metabolic phenotyping in health and disease. Cell. 2008;134(5):714-7.

11. Chen L, Cheng CY, Choi H, Ikram MK, Sabanayagam C, Tan GS, Tian D, Zhang L, Venkatesan G, Tai ES, et al. Plasma Metabonomic profiling of diabetic retinopathy. Diabetes. 2016;65(4):1099-108.

12. Burgess LG, Uppal K, Walker DI, Roberson RM, Tran V, Parks MB, Wade EA, May AT, Umfress AC, Jarrell KL, et al. Metabolome-wide association study of primary open angle Glaucoma. Invest Ophthalmol Vis Sci. 2015;56(8):5020-8.

13. Chylack LT Jr, Wolfe JK, Singer DM, Leske MC, Bullimore MA, Bailey IL, Friend J, McCarthy D, Wu SY. The Lens opacities classification system III. The longitudinal study of cataract study group. Arch Ophthalmol. 1993;111(6): 831-6.

14. Barbas-Bernardos C, Armitage EG, Garcia A, Merida S, Navea A, Bosch-Morell F, Barbas C. Looking into aqueous humor through metabolomics spectacles - exploring its metabolic characteristics in relation to myopia. J Pharm Biomed Anal. 2016:127:18-25.

15. Trygg J, Holmes E, Lundstedt T. Chemometrics in metabonomics. J Proteome Res. 2007;6(2):469-79.

16. Trygg J, Wold S. Orthogonal projections to latent structures (O-PLS). J Chemometrics: J Chemometrics Soc. 2002;16(3):119-28.

17. Kanehisa M, Goto S, Sato Y, Kawashima M, Furumichi M, Tanabe M. Data, information, knowledge and principle: back to metabolism in KEGG. Nucleic Acids Res. 2014;42(Database issue):D199-205.

18. Chong J, Soufan O, Li C, Caraus I, Li S, Bourque G, Wishart DS, Xia J. MetaboAnalyst 4.0: towards more transparent and integrative metabolomics analysis. Nucleic Acids Res. 2018;46(W1):W486-94.

19. Agudo-Barriuso M, Lahoz A, Nadal-Nicolas FM, Sobrado-Calvo P, Piquer-Gi M, Diaz-Llopis M, Vidal-Sanz M, Mullor JL. Metabolomic changes in the rat retina after optic nerve crush. Invest Ophthalmol Vis Sci. 2013;54(6):4249-59.

20. Edwards G, Aribindi K, Guerra Y, Bhattacharya SK. Sphingolipids and ceramides of mouse aqueous humor: comparative profiles from normotensive and hypertensive DBA/2J mice. Biochimie. 2014;105:99-109.

21. Wishart DS, Feunang YD, Marcu A, Guo AC, Liang K, Vazquez-Fresno R, Sajed T, Johnson D, Li C, Karu N, et al. HMDB 4.0: the human metabolome database for 2018. Nucleic Acids Res. 2018;46(D1):D608-17.

22. Valenciano Al, Mayordomo R, de La Rosa EJ, Hallbook F. Biotin decreases retinal apoptosis and induces eye malformations in the early chick embryo. Neuroreport. 2002:13(3):297-9.

23. Benoist d'Azy C, Pereira B, Chiambaretta F, Dutheil F. Oxidative and antioxidative stress markers in chronic glaucoma: a systematic review and meta-analysis. PLoS One. 2016;11(12):e0166915.

24. Fernandes CG, Borges CG, Seminotti B, Amaral AU, Knebel LA, Eichler $P$, de Oliveira AB, Leipnitz G, Wajner M. Experimental evidence that 
Methylmalonic acid provokes oxidative damage and compromises antioxidant defenses in nerve terminal and striatum of young rats. Cell Mol Neurobiol. 2011;31(5):775-85.

25. AlOwain M, Khalifa OA, Al Sahlawi Z, Hussein MH, Sulaiman RA, Al-Sayed M, Rahbeeni Z, Al-Hassnan Z, Al-Zaidan H, Nezzar H, et al. Optic neuropathy in classical methylmalonic acidemia. Ophthalmic Genet. 2019;40(4):313-22.

26. Alvarez LM, Jameson E, Parry NRA, Lloyd C, Ashworth JL. Optic neuropathy in methylmalonic acidemia and propionic acidemia. Brit J Ophthalmol. 2016;100(1):98-104.

27. Pinar-Sueiro S, Martinez-Fernandez R, Lage-Medina S, Aldamiz-Echevarria L, Vecino E. Optic neuropathy in methylmalonic acidemia: the role of neuroprotection. J Inherit Metab Dis. 2010;33:S199-203.

28. Thakur K, Tomar SK, Singh AK, Mandal S, Arora S. Riboflavin and health: a review of recent human research. Crit Rev Food Sci Nutr. 2017;57(17):3650-60.

29. Klein BE, Klein R, Lee KE. Diabetes, cardiovascular disease, selected cardiovascular disease risk factors, and the 5-year incidence of age-related cataract and progression of lens opacities: the beaver dam eye study. Am J Ophthalmol. 1998;126(6):782-90.

30. Varma SD, Schocket SS, Richards RD. Implications of aldose reductase in cataracts in human diabetes. Invest Ophthalmol Vis Sci. 1979;18(3):237-41.

31. Reinehr S, Reinhard J, Gandej M, Kuehn S, Noristani R, Faissner A, Dick HB, Joachim SC. Simultaneous complement response via Lectin pathway in retina and optic nerve in an experimental autoimmune Glaucoma model. Front Cell Neurosci. 2016;10:140.

\section{Publisher's Note}

Springer Nature remains neutral with regard to jurisdictional claims in published maps and institutional affiliations.

Ready to submit your research? Choose BMC and benefit from:

- fast, convenient online submission

- thorough peer review by experienced researchers in your field

- rapid publication on acceptance

- support for research data, including large and complex data types

- gold Open Access which fosters wider collaboration and increased citations

- maximum visibility for your research: over $100 \mathrm{M}$ website views per year

At BMC, research is always in progress.

Learn more biomedcentral.com/submissions 